Rev. Fac. Med. vet. Zootec. Univ. S. Paulo, $13(2): 333-8,1976$

\title{
DESALEITAMENTO PRECOCE DE BEZERROS III. DESENVOLVIMENTO DO RÚMEN
}

Carlos de Sousa LUCCI.

\begin{abstract}
RFMV-A 24
LUCCI, C. DE S. Desaleitamento precoce de beaerros. III. Desenvolvimento do rumen. vet. Zootec. Univ, S. Paulo, 13(2):333-8, I976.

RESUMo: Seis bezerros recebendo raçóes iniciais sem feno incorporado, com $12 \%, 15 \%$ e $18 \%$ de $P . D .$, e com $25 \%$ de feno incorporado e $12 \% .15 \%$ e $18 \%$ de P. D. foram conduzidos até os 91 dias de idade sob controle semanal dos pesos vivos e perimetros abdominais e controle diario do consumo de alimentos. Com 92 dias de idade foram abatidos para observaçäo do desenvolvimento de seus ventrículos gástricos. Näo surgiram evidéncias que os pesos dos conteúdos dos tratos digestivos dos bezerros colocados nos diversos tratamentos fossem diferentes.
\end{abstract}

UnITERmos: Bezerros"; Desaleitamento precoce"; Rümen desenvolvimento*

\section{INTRODUCAO}

$\mathrm{Na}$ alimentação de ruminantes, o capítulo referente aos bezerros é por certo o que mais evoluiu nas últimas décadas, graças aos novos conhecimentos sobre fisiologia e desenvolvimento do rimen em idade precoce e sobre sua população microbiológica, em relação a várias diétas empregadas. Em nosso meio criatório, somente há poucos anos surgiu interesse na aplicação de novas técnicas de alimentar bezerros, e conse quentemente a preocupação dos nutricionistas brasileiros em adaptar dados de pisquisas estrangeiras, obtidos sob outras condiçōes de exploração animal. O objetivo deste trabalho foi observar possiveis diferenças no desenvolvimento e proporções dos ventrículos gástricos de bezerros submetidos a dietas diferentes.

PRESTON 11 levantou a hipótese que o desenvolvimento do rúmen depende menos da jdade que da precocidade do ato de ingerir alimentos sólidos.

LENGE7MANN e ALLEN'n coletando material do rúmen de bezerros que recebiam feno e concentrados, encontrou ampla variação em suas características físicas até 2 meses, mas em maiores idacles houve uniformidade quanto a cor, odor e grau de maceração.

BROWNLEE 1 fornecendo dietas com feno, ou gramineas, ou concentrados para bezerros, notou papilas medindo respec-

\footnotetext{
- Professor Livre Docente.

Departamento de Produção Animal da Faculdade de Medicina Veterinária e Zootecnica da USP.
} 
tivamente $5 \mathrm{~mm}, 7 \mathrm{~mm}$ e $8 \mathrm{~mm}$ de altura, abatendo os animais com 12 simanas. Os pesos dos rumens foram $1056 \mathrm{~g}, 1448 \mathrm{~g}$ e $1454 \mathrm{~g}$.

WARNER, FLATT e LOOSLI" $"$ ob servaram que dietas contendo feno provocavam aumento na capacidade dos proventrículos.

SWANSON e HARRIS 13 em estudo com animais recebendo ração inicial e feno. sacrificou um bezerro que não ruminava e trés que ruminavam, encontrando no rúmen do primeiro palha em pedaços grosseiros, poucos grãos e muito líquido. Os três que ruminavam apresentaram conteúdos ruminais finamente macerados.

FLATT, WARNER \& LOOSI 2 afirmam que os critérios mais sensiveis para medir os efeitos das várias dietas sobre o desenvolvimento do rúmen sāo as medições das papilas da mucosa desse orgão e de sua capacidade de volume.

GODFREY 3 para bezerros de 12-14 semanas de idade forneceu os rasultados scguintes: $1,150 \mathrm{~kg}$ a $1,375 \mathrm{~kg}$ de peso de I'imen-retículo e 0,595 a $0,700 \mathrm{~kg}$ para omaso-abomaso. A relação em peso I ümen-reticulo/cmaso-abomaso atingiu 65 : $35 \mathrm{~cm} 6$ semanas.

NOLLER, DICKSON e HILL 9 recomendaram a inclusão de feno na ração inicial além de fornecer o feno a parte, como meio de se evitar formação de placas e eresōes nas mucosas dos rúmens.

STOBO, ROY e GASTON 12 fornecendo proporções diferentes de ração inicial e feno, encontraram nos tratamentos com maiores proporçōes de concentra. dos, bezerros cujas mucosas de rúmen possuiam papilas mais altas. Os AA relacionaram alturas de papilas com desempenhos dos animais.

HUBER 4 afirma que a introdução de alimentos sólidos na dieta de bezerros faz com qua as papilas atinjam um má. ximo de altura ( 3 a $7 \mathrm{~mm}$ ) já $\operatorname{com} 8$ scmanas de idade. Dos alimentos sólidos, os concentrados seriam os responsáveis p?lo pleno desenvolvimento das papilas, pela libcração de ácidos graxos butírico, propiônico e acético, citados na ordem de eficiência.

JAHN, CHANDLER e POLAN 5 for. neccndo para bezerros raçōes com por. centageas crescentes de palhas notaram aumento dos conteúdos dos tratos diges. tivos dos animais, nos niveis mais elevaJos, como também um aumento no teor de ácich acético em detrimento do propiônico.

LUCCI 7 e PAIVA LUCCI 10 estu. daram ventrículos gástricos de bezerros abatidos com 13 e 20 semanas de idade, submetidos a dietas de desaleitamento precoce $\theta$ apresentaram dados de pesos c proporções rúmen-reticulo/omaso-abo. maso.

\section{MATERIAL E MÉTODOS}

As observações presentes foram conduziclas no Centro de Zootecnia e Indústrias Pecuárias "Fernando Costa", em Pirassuninga, S.P., em 1973. Foram usados seis bezerros intigrantes de um dos blocos experimentais de estudo (LUCCI ${ }^{8}$ ) onde se comparavam 6 tratamentos em um arranjo fatorial $2 \times 3$ : raçōes $\mathrm{A}$ e $\mathrm{B}$, respectivamente, sem fano e com $25 \%$ de feno incorporado, versus 3 niveis de P.D.: $" 1 "=12 \% ; " 2 "=15 \%$ e " $3 "=18 \%$. Feno à vontade foi constante em todos os tratamentos.

Foram tomadas mediçōes de perímetro abdominal a cada 7 dias, dos 14 aos 91 dias de idade. Os consumos de ração inicial e feno eram controlados diariamente. Os scis animais foram sacrificados ao completarem 92 dias de idade, sempre pela manhã, no mesmo horário. Imediatamcnte após o sacrifício feito com os bezerros em decúbito lateral com 
- fim de evitar-se possiveis regurgitações e perdas de material do rumen, retirou se os ventriculos do aparelho digestivo, praticando-se para isso ligaduras na porção terminal do esôfago, entre retículo e omaso e entre abomaso e primeras porções do duodeno logo após o esfincwr pilórico.

Os ventriculos retirados foram pesados com seus conteüdos, considerando-se as porções rúmen-retículo e omasoabomaso. Em seguida, separou-se o rimen-retículo o qual foi pesado com szus conteúdos. O peso do omaso-abo. maso mais conteúdos foi obtido por diferença. Finalmente, praticou-se incisão do rumen-reticulo e do omaso-abomaso, livrando-se de saus conteúdos, e após cuidadosa lavagem para retirada dos resíduos alimentares, foram pesados os tecidos (continentes). Antes dessa pesagem, tomou-se o cuidado de espremer os tecidos com as mãos deixando sa escorrer excessos de água. Por diferença, foram calculados os pesos dos conteúdos do rúmen-retículo e do omaso-abomaso.

A mucosa do rúmen foi examinada e as papilas foram medidas em suas alturas, com auxílio de um paquímetro, tomando-se 5 leituras ao acaso, de um corte de $10 \mathrm{~cm}$ do saco anterior dorsal do rúmen.

Observações paralelas sobre aspectos do conteúdo ruminal. sua coloração odor e grau de maceração, também foram executadas.

\section{RESULTADOS}

A Tabela 1 fornece pesos vivos finais dos 6 bezerros, ganhos de peso, ganhos em perímetros abdominais e consumos de ração inicial, feno de alfafa (ofertados à vontade), concentrados, feno total (das raçöes iniciais $\mathfrak{B}+$ feno à vontade), $e$ matéria seca, no periodo de 14 a 91 dias de vida.

TABELA 1 - Pesos finais e ganhos de peso, em quilogramas, ganhos em perimetros abdominais, em $\mathrm{cm}$, e consumos de alimentos, em quilogramas, curante o periodo de 14 a 91 dias de idade.

\begin{tabular}{|c|c|c|c|c|c|c|}
\hline \multirow{2}{*}{ OBSERVAÇOES } & \multicolumn{6}{|c|}{ TRA TA M E N T OS } \\
\hline & A1 & A2 & A3 & $\mathbf{B 1}$ & $\mathbf{B 2}$ & $\mathbf{B 3}$ \\
\hline Pesos finals (kg) & 64,9 & 60.4 & 88,4 & 65.1 & 58,5 & 72,7 \\
\hline Ganhos de peso $(\mathrm{kg})$ & 31.5 & 25.3 & 46.0 & 30,1 & 20.1 & 37,2 \\
\hline Perimetros abdominais $(\mathrm{cm})$ & 33.0 & 26.0 & 46.0 & 40.0 & 47.0 & 47.0 \\
\hline Raçăo Inicial (kg) & 73.5 & 68.7 & 106.6 & 31.8 & 75.8 & 129.4 \\
\hline Feno (kg) & 9.6 & 6.1 & 10,5 & 9.5 & 2.9 & 3,0 \\
\hline Feno total $(\mathrm{kg})$ & 9.6 & 6.1 & 10,5 & 32.4 & 21,8 & 35.4 \\
\hline Concentrados $(\mathrm{kg})$ & 73.5 & 68.7 & 106.6 & 68.8 & 56.9 & 97,1 \\
\hline M.S. $(\mathrm{kg})$ & 85,2 & 76.5 & 116,4 & 102,0 & 80.1 & 130.4 \\
\hline
\end{tabular}

A Tabela 2 forneca consumos de raçāo inicial, feno, feno total, concentra- dos e M.S., durante a última semana de vida dos 6 bezerros. 
TABELA 2 - Consumo de alimentos em quilogramas, durante a última semana de vida dos bezerros.

\begin{tabular}{|c|c|c|c|c|c|c|}
\hline \multirow{2}{*}{ OBSERVAÇÖES } & \multicolumn{6}{|c|}{ TRATAMENTOS } \\
\hline & Al & A2 & A3 & $\mathbf{B} 1$ & B2 & B3 \\
\hline Raçáo intcial (kg) & 16.3 & 14.2 & 15.7 & 17,2 & 13.7 & 17,4 \\
\hline Feno $(\mathrm{kg})$ & 2.4 & 1,3 & 4.5 & 1.4 & 0.1 & 1.4 \\
\hline Feno total $(\mathrm{kg})$ & 2.4 & 1,3 & 4.5 & 5.7 & 3.5 & 5,8 \\
\hline Concentrados (kg) & 16.3 & 14,2 & 15,7 & 12.9 & 10.3 & 13.0 \\
\hline M.S. $(\mathrm{kg})$ & 16,5 & 13,7 & 18,1 & 16.4 & 13,3 & 16,8 \\
\hline
\end{tabular}

Os conteúdos dos rúmens de todos os animais apresentaram-se finalmente macerados e homogêneos. A coloração cios conteúdos ruminais foi amarelo claro para os bezerros dos tratamentos Al,
A2 e B2; esvercleados para os bezerros dos tratamentos A3, B1 e B3.

A Tabela 3 fornece resultados de exames do aparelho digestivo.

TABELA 3 - Resultados de exames do aparelhos digestivo de bezerros, sacrificados aos 92 dias de vida.

\begin{tabular}{|c|c|c|c|c|c|c|}
\hline \multirow{2}{*}{ OBSERVAÇOES } & \multicolumn{6}{|c|}{$T R A T A M E N T O S$} \\
\hline & Al & A2 & A3 & B1 & $\mathbf{B 2}$ & B3 \\
\hline RROA. chelos (kg) & 7.830 & 8.820 & 10.570 & 7.620 & 10,370 & 10.100 \\
\hline RR, chelos $(\mathrm{kg})$ & 6.320 & 6.340 & 8.170 & 5.730 & 8.480 & 8,200 \\
\hline OA. chelos (kg) & 1.510 & 2,480 & 2,400 & 1.890 & 1.890 & 1,900 \\
\hline RROA. vaztos (kg) & 1.800 & 1,990 & 2,760 & 1.740 & 1,745 & 2.000 \\
\hline RR, vazlos (kg) & 1.190 & 1.240 & 1,620 & 1.000 & 1,100 & 1.210 \\
\hline OA, vazios (kg) & 0.610 & 0.750 & 1,140 & 0.740 & 0.645 & 0.790 \\
\hline RROA, conteudo $(\mathrm{kg})$ & 6.030 & 6.830 & 7.810 & 5,880 & 8.625 & 8,100 \\
\hline$R R$, conteúdo $(\mathrm{kg})$ & 5.130 & 5.100 & 6.550 & 4.730 & 7.380 & 6.990 \\
\hline OA, conteúdo $(\mathrm{kg})$ & 0.900 & 1.730 & 1,260 & 1.150 & 1,245 & 1,110 \\
\hline Altura papllas (mm) & 8.5 & 6.6 & 8.4 & 7.2 & 5.2 & 8.7 \\
\hline RR/ ( $\%$ em peso RROA) & 66.1 & 62.3 & 58,7 & 57.5 & 63.0 & 60.5 \\
\hline
\end{tabular}

$\mathbf{R R}$ - rúmen-reticulo: DA - omaso-abomaso: RROA - rúmen-retículo + omaso-abomaso

\section{DISCUSSAO}

No presente estudo, os conteúdos dos rumens apresentaramse sempre bem macerados e homogêneos que, segundo LENGEMAN e ALLEN ' e SWANSON e HARRIS 13 comprovou a eficiència da ruminação por parte dos bezerros.
A cor esverdeada obtida nos conteúdos ruminais em $\mathbf{A} 3, \mathbf{B} 1$ e $\mathbf{B} 3$, podem ser explicadas pelo maior consumo de feno total nesses casos durante os últimos dias de vida. (Tabela 2).

O desenvolvimento das papilas (Tabela 3) foi evidente em todos os casos, 
mas inferior em A2 e B2. quando ocorrou consumo notavelmente menor de M.S., na última semana. Isso concorda com as afirmativas de PRESTON 11, FLATT, WARNER e LOOSI 2 e STOBO, ROY e GASTON 12, que o pleno desenvolvimento do rúmen está relacionado ao consumo de M.S. proveniente de alimentos sólidos.

Segundo FLATT, WARNER e LOOS. II 1", HUBER 4 e STOBO, ROY e GASTON 12 , as papilas se desenvolvem mais pelos produtos de fermentação que pela natureza física grosseira dos alimentos. No presente caso, as papilas foram maiores quando ocorerram ingestōes mais elevadas de M.S., não havendo relação aparente com o consumo de concentracos.

Os pesos dos rúmen-retículos vazios, com exceção dos bezerros A3 foram concordes com os valores apresentados por GODEREY 3, LUCCI 7 e BROWNLEE 1, e a relação de peso rúmen-retículo/omasoabomaso com os valores de GODFREY 3 e LUCCI 7 .

Os conteúdos de rúmen-retículos foram mais pesados no bezerro submetido ao tratamento B2, que ingeriu menos M.S. na semana anterior (Tabelas 2 e 3 ). ccorrendo portanto uma velocidade de trânsito diminuida através daqueles orgãos. Os pesos obtidos para os conteúdos concordam com aqueles fornecidos por LUCCI 7 e PAIVA e LUCCI 10 em die. tas semelhantes.

Não surgiram evidências que os conteúdos dos tratos digestivos em "B", cujas raçōes continham $25 \%$ de feno incluido, fossem maiores que em " $\mathrm{A}$ ", sem feno, como sugariram os trabalhos de JAHN, CHANDLER e POLAN 5 e e WARNER, FLATT e LOOSLI 1*. Tam. bém nāo foram encontradas placas, sedimentação de resíduos alimentares ou erosāo de mucosa nos rúmens dos bezerros, não se justificando as recomentaçōes de NOLLER, DICKSON e HILL9 de que o feno deve ser incluido na ração inicial além de fornecido à vontade, para evitar-se aqueles problemas.

Maiores pesos dos quatro ventrículos repletos, ou do rúmen-reticulo repleto, bem como maiores pesos dos conteúdos do rúmen-retículo ou do total dos quatro ventrículos, foram encontrados nos bezerros em A3, B2 e B3 (Tabela 3), justamente os animais que apresentaram maiores ganhos de perímetro abdominal (Tabela 1), sugerindo uma relação entre essas varávieis.

\section{CONCLUSŌES}

Nas condições do experimento, foi possivel emitir as seguintes conclusões:

1 - Não surgiram evidências que os pesos de conteúdos dos tratos digestivos de bəzerros, colocados em tratamento com raçōes iniciais contendo ou não $25 \%$ de feno incluido, fossem diferentes.

2. - A ausência de feno nas rações iniciais não implicou em surgimento de problemas na mucosa do rúmen, como placas, sedimentações de detritos ou erosōes.

3 - Houve uma aparente relação entre ganhos em perimetros abdominais e pesos de continente e conteúdo ou apenas conteúdo dos rúmen-reticulos 
Lucci, C. DE S. Early weaning of calves. III. Rumen development. Rev. Fac. Med. Rev. Fac. Med. vet. Zootec. Univ. S. Paulo, 13(2):333-8, 1976.

SumMary: Six calves fed with calf starters a) without hay e b) with hay included, each one taking three levels as P. D.: $1-12 \%: 2=15 \sigma_{\circ}^{\circ}$ and 3 $=18 \%$ were controled weekly from 14 to 91 days old for paunch birth gains and daily for feed consumption. At 92 days old the calves were slawghtered to observe their rumen development. The weights of rumen contents at all theatments were. not differents.

UNITERMS: Calves*; Restricted milk weaning system*; Rumen, development*.

\section{REFERENCIAS BIBLIOGRÁFICAS}

1 - BROWNLEE. A. The development of ritmen paplline in cattle fed on different dlets. Brit. vet. J., $112(9): 369-75$, 1958.

2 - FLATT, W. R.: WARNER. R. G.: LOOSLI. J. K. Evaluation of several techniques used in the study of developing rumen function. Ithaca, 1959. 30p. (Cornell University Agric. Exp. Sta.).

3 - GODFREDY. N. W. The funcional development of the calf. I. Growth of the stomach of the calf. J.agric. Sci., Camb., 57(2):173-6. 1961.

4 - HUBER. J. T. Symposium on calf nutrition and rearing. Development of digestive and metabolic apparatus of the calf. J. Dairy Sci., 52(8):1303-15. 1069.

5 - JAHN, E.: CHANDLER, P. T.: POLAN, C. E. Erfects of fiber and ration of starch to sugar on performance or rumlnating calves. J. Dairy Sci., 53 (4) : 466-74, 1970

6 - LENGEMANN, F. W. \& ALLEN. N. N. The jeveloping of rumen function in the dairy calf. I. Some characteristics of the rumen contents of cattle of varous ages. J. Dairy Sci., 38(6):651-56, 1955.

7 - ILUCCI, C. de S. Estudo sobre o efeito cie diferentes niveis de flbra bruta em raçōes de desmama precoce de bezerros de racis lelteiras. Bol. Ind. Anim., 39(1):67-149, 1972.
8 - LUCCI. C. de S. Desaleltamento precoce de bezerros. I. Nivels de proteina e energla. Rev. Fac. Med. vet. Zootec. Univ. S. Paulo, 13(2): 1976.

9 - NOLLEF. C. H.: DICKSON. I. A.; HILI. D. L. Valve of hay and rumen Inoculation in an early weaning system for dairy calves. J. Dairy Sci, 45:197201, 1962.

10 - PAIVA. J. A. J. \& LUCCI. C. de S. Alimentacăo de bezerros com mistura concentrada comum mais feno de soja perene. II. Desenvolvimento dos proventrículos. Bol. Ind. Anim., 29 (1) :151-9, 1972 .

11 - PRESTON, T. R. Dry feeding of calves. A firicultute, G2(10):462-6, 1956.

12 - STOBO. I. J. F.: ROY, J. H.: GASTON. H.J. Rumen development In the calf. I. The effect of diets conteining different proportlons of concentrates to hay on rumen development. Brit. J. Nutr., 20:171-88, 1966.

13 - SWANSON, E. W. \& HARRIS JR. J.D Development of rumination in the young calf. J. Dairy Sci., $\$ 1$ (12):176876. 1958.

14 - WARNER. R. G.: FLATT. W. P.: LOOSII, I. K. Dletary factors influencling the development of the ruminant stomach. J.agric. Fd. Chem., 4(9):788. 92. 1956.

Recebido para publicaçāo em 27-7-76 Aprcvado para publlcaçăo em 13-9-73 